\title{
Design of the Uyghur FrameNet Desktop
}

\author{
Mirejiguli Rezhake and Alifu Kuerban
}

\begin{abstract}
This paper presents a case study aimed at adapting FrameNet Desktop to Uyghur FrameNet. On the example sentence of the lexical unit دئانه قیى (donate), gives a detailed overview of the annotation tasks and process within FrameNet Desktop, which illustrates many functions of the tool. In what follows, we presents lexical unit report and lexical entry report, which are automatically generated based on annotation completed using the annotaion Desktop.
\end{abstract}

Index Terms-Uyghur, Uyghur FrameNet, annotation Desktop.

\section{INTRODUCTION}

The Berkeley FrameNet project has developed a lexical knowledge base provided background and the power for the existence and using of words and a number of semantically and syntactically annotated sentences [1]. Suceessfully, it has also been applied to various natural language processing such as question answering systems, information retrieval and machine translation Etc.

Comparing with languages such as English, Chinese, Arabic-based Uyghur semantic analysis research and related methods is rare and lagging, is still in the primary state of exploration. Aim at the current state of Uyghur semantic analysis research is not mature and semantic role labeling is bottlenecks, we chose the Fillmore semantics as theoretical basis, with ideas of the Berkeley FrameNet and the Chinese CFN design system for reference, combined Uyghur source language frame semantics description system, we constructed a word level Uyghur FrameNet (henceforth, UFN) .

As same with the FrameNet in other languages such as English, Chinese, UFN has three major components: frame database, lexical database and annotated example sentences [2]. Frame database as a frame to unit, clearly presents a) frame definition and b) semantic roles and corresponding example sentences, and c) describes concept relationships between the frame and other frames. annotated example sentences contains lots of sentences with frame semantic and syntactic tagging information which would be automatically generated based on annotation completed using UFN Desktop annotation tools. The lexical database contains a) formulas which capture the syntactic ways in which elements of the semantic frame can be realized within the phrases or sentences built up around the world; b) links to semantically

Manuscript received February 15, 2014; revised April 18, 2014. This work was supported by China Natural Sciences. Foundation project subsidization (authorized number 61163029) and Xinjiang Uyghur autunomous region university scientific research project (authorized number XJEDU2011I107).

Mirejiguli Rezhake and Alifu Kuerban are with the Information Science and Engineering Institute of Xinjiang University, Urumqi, China (e-mail: mirajigul123@sina.com, Ghalipk@xju.edu.cn). annotated example sentences which illustrate each of the potential realization patterns identified in the formula.

Construction of UFN is a huge project, Regardless of a) entering and editing frame descriptions and lists of lexical units, b) extracting sentences from the corpus, c) annotating selected sentences with frame-relevant labels, or d) organizing and displaying the results are need to highly human-computer interactive tools. This paper discussed the design and implementation of the Uyghur Framenet desktop tool.

\section{UYGHUR LANGUAGE}

Uyghur language is main communication tool of Uyghur people. It is one of the statutory working languages of Xingjiang Uyghur Autonomous Region, China and common language of other ethnic minorities in Xingjiang for communication as well.

Uyghur belongs to the Turkic language family of the Altaic language system. At present, Uyghur is written in Arabic scripts with some modifications. As same with any kind of language, An Uyghur sentence constituted by words, in which words separated by space or punctuation marks. As an agglutinative language, the structure of Uyghur word is "prefix+stem+suffix $1+$ suffix $2+\ldots$... Because Uyghur has its own characters and nature, It needs approaching some different methods in processing.

The research on the Uyghur information processing in the 20th century can be dated back to the beginning of $1980^{\circ} \mathrm{s}$. Up to now, Uyghur language processing work obtained some promising results. However, comparing with Europe language and Chinese, it still needs a lot of work to be done Especially on semantic studies of information processing still does not have its own theoretical system. In this paper, we have designed "Uyghur FrameNet Desktop" for the first time in order to meet the urgent requirement of the current semantic research. The result of this paper, have high value on semantic research and application development of Uyghur even the other languages of Altaic.

\section{UFN DESKTOP ANNOTATION TASK}

Initially, the FrameNet Desktop was designed for work on English. Scholars around the world have recognized that it can be across language used and have general value. Since then, it has been adapted by various sites setting up FrameNets for different languages, such as German, Japanese, Spanish and Chinese.

However, there is a difference between languages the way of lexicalization, therefore, the frame and the frame elements need to be a modified according to the characteristic of different languages. English and Uyghur are quite differ from 
structure and knowledge representation, so we make more efforts on modifying the FrameNet Desktop [3] and adjust them in accordance with Uyghur characteristics. Computer-aided annotation of example sentences, the goal is to document the range of semantic and syntactic combinatory possibilities valences of each word in each of its senses.

The Berkeley FrameNet project annotation sentences on layers, and mapping from semantic meaning to syntactic expressions can be realized easily [3]. Similar approach has been taken for Uyghur sentence annotation in UFN Desktop. For a given sentence, target word and it is semantic frame, annotate to direct dependents of target words, and do not annotate constituents that are understood only through context as referring to the filler of a particular frame element role [3]. These dependents are annotated, on their separate layers, for frame element name, grammatical function (GF), and phrase type (PT) and these three types; for example:

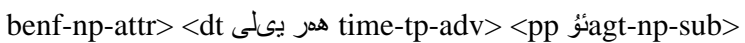

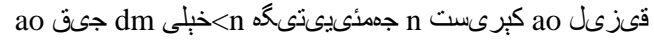

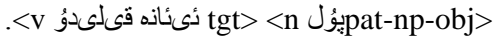

(Every year he donates a lot of money to the Red Cross Society)

In above sentence, target بـىئانه قىل belongs to بمرمك (giving) frame, using "tgt" lables annotate to target; agt (agent), time, benf (benifector) and pat (patient) are semantic role lables; np(noun phrase), tp (time phrase) are phrase type lables ;sub (subject) ,adv (adverbial) , attr(attribute) obj (object) are syntactic function labels. Pp (personal pronoun), dt (time adverbs), ao (positive adjective), n (noun), dm (state adverbs) and v (verb) are part-of-speech lables.

It is known from the above analysis, UFN sentence labeled objects as the target word, frame elements, phrase type, syntactic function and part-of-speech. Below, we discuss relative terms.

\section{A. Target Word}

UFN is lexical resource for Uyghur, based on frame semantics and supported by corpus evidence. Frame semantics believe that, frame is the background and motivation of word understanding, In UFN, Uyghur verbs, adjectives, and in the event noun can act as a frame. A frame can contain more than one target word, that is, a frame that can be activated multiple target word. When we annotate an example sentence, we always do so from the point of view one particular target word in the sentence.

\section{B. Frame Element}

Frame element is a frame participant and the support which is a concept role presented in the scheme scene. The frame element describes the semantics and the syntactic features of the frame element with corresponding demonstration. It carries on the concise description, enables the sentence to be understood, and supplies the regular information for text labeling. In the different frame the numbers and the concept roles of frame are different due to the cognitive background of frame element [2]. The frame elements may be divided into the core and non-core frame elements according to participation and the support function of frame element. A core frame element is one that instantiates a conceptually necessary component of a frame, and must be showing the properties of the frame. Non-core frame element can't show any individual character of a frame, and it can occur in most frames.

In the UFN corpus, some core frame elements are conceptually necessary but do not occur as lexical or phrasal material; these we call null instantiations. There are three types of null instantiations-constructional, definite and indefinite. We will now briefly discuss the three types of omission. Constructional Null Instantiation (CNI) is licensed by a grammatical construction, as with the missing subject of an imperative sentence, which does not depend on the identity of any particular lexical item [3]. For example, in the Giving frame, all lexical units happen to allow the core frame element donor to be omitted under constructional null instantiation. This is shown for lexical unit Donate in following sentence 1): With Definite Null Instantiation (DNI) the oMissing core frame element must be understood in the linguistic or discourse context [3]. In sentence 2) below, in the Giving frame, the recipient in not expressed overtly in the sentence, but it is understood from the context. Indefinite null instantiation (INI) covers missing objects of certain transitive verbs that are characterized as used intransitively; the missing of object of such a verb often has a special interpretation [3]. In sentence 3) below, in the Giving frame, the missing object of give is most likely to be audience.

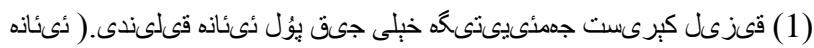

$$
\text { قولغؤجى (1) }
$$

(Lots of money donated to the Red Cross Society. )

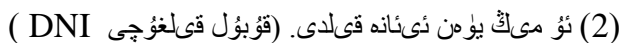

$$
\begin{aligned}
& \text { (He donated \$thousand.) }
\end{aligned}
$$

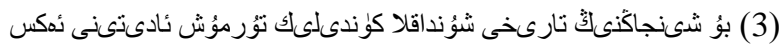

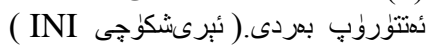

(It gives an overview of Xinjiang's history and its daily life and tradition.)

\section{Phrase Type (PT)}

Phrase is a group or words that express a concept and is used as a unit within a sentence. In the UFN, Phrase is a broad concept; it may be composed of a single Uyghur word or more than two words. In annotation, each constituent tagged with a frame element in respect to a target word is assigned a phrase type tag in respect to that target.

\section{Grammatical Function (GF)}

The grammatical functions (GF) that are assigned describe the ways in which the constituents satisfy abstract grammatical requirements of the target word. In annotation, only constituents tagged with frame elements are assigned grammatical functions.

\section{ANNOTATION PROCESS}

The UFN Desktop software has deployed that combines and runs on c\# (2.0) application system using SQL2008 for data storage. We now turn to UFN Desktop annotation process. Specific process is shown in Fig. 1. In annotation, 
our main focus is on the goal of recording the range of semantic and syntactic combinatory possibilities (valences) of each word in each of its senses. To that end, we extract sentences from the different texts of a corpus. Then we annotate a selection of the extracted sentences in respect to that particular LU. Once annotaion is completed, we can examine the semantic and syntactic patterns.

FrameNet annotations are constellations of triples that make up the frame element realization for each annotated sentence, each consisting of a frame element, a grammatical function and a phrase type.

On the basis of the above tasks, the semantic and syntactic Valency pattern of a lexical unit generated automatically in the annotated sentences database. The above example sentence takes the follosing patterns:

Semantic pattern(read right to left):

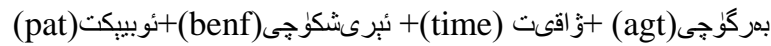
Syntactic pattern (read right to left):

noun phrase+Noun phrase+data phrase+noun phrasetp

[object] [attribute] [adverbial] [subject]

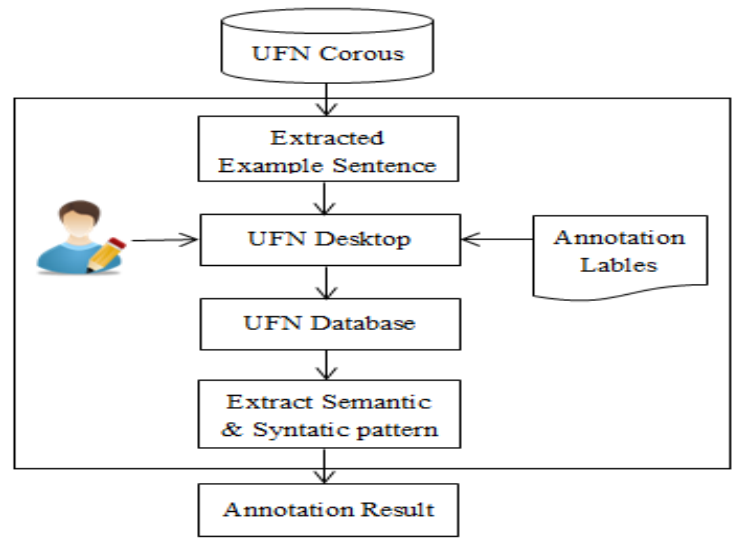

Fig. 1. UFN desktop annotation process.

\section{THE IMPLEMENTATION OF THE UFN DESKTOP}

The system design for UFN annotator, aim at reduce the format and spelling mistakes problems in the process of annotation, realized the lexicon automatically generated, and presentation lexical unit and lexical entry report.

UFN Desktop annotation window as shown in Fig. 2, divided into four workspace: workspace 1) is frame list, contains an expanding list that gives direct access to the main kinds of objects, such as frame, frame elements, and lexical units; workspace 2) is selecting sentences; workspace 3) is annotation layers; workspace 4) is lists of labels available for each of the annotation layers.

Once annotator chose a lexical unit for annotation, a list of sentences appear in the workspace 1). annotator read through the sentences and select a with variety information of semantic and syntactic pattern sentence for annotation. Selected sentence appear in the workspace 2), and which contains 7 seven annotation layers are target, frame element, phrase type, grammatical function, other, sentence and part of speech layers. through mouse dragging annotator select target and its direct dependents, and then use conrresponding lables as [4]-[6] to filling each of layers in the workspace 3).
After annotation is complete, through review the lexical unit report and lexical entry report we can exammine annotated result and valence patterns they manifested. these reports are automatically generated based on annotation completed using UFN Desktop annotation tools. The two parts of this report are illustrated in Fig. 3 and Fig. 4, respectively, for lexical unit ئى قئنه قىل.

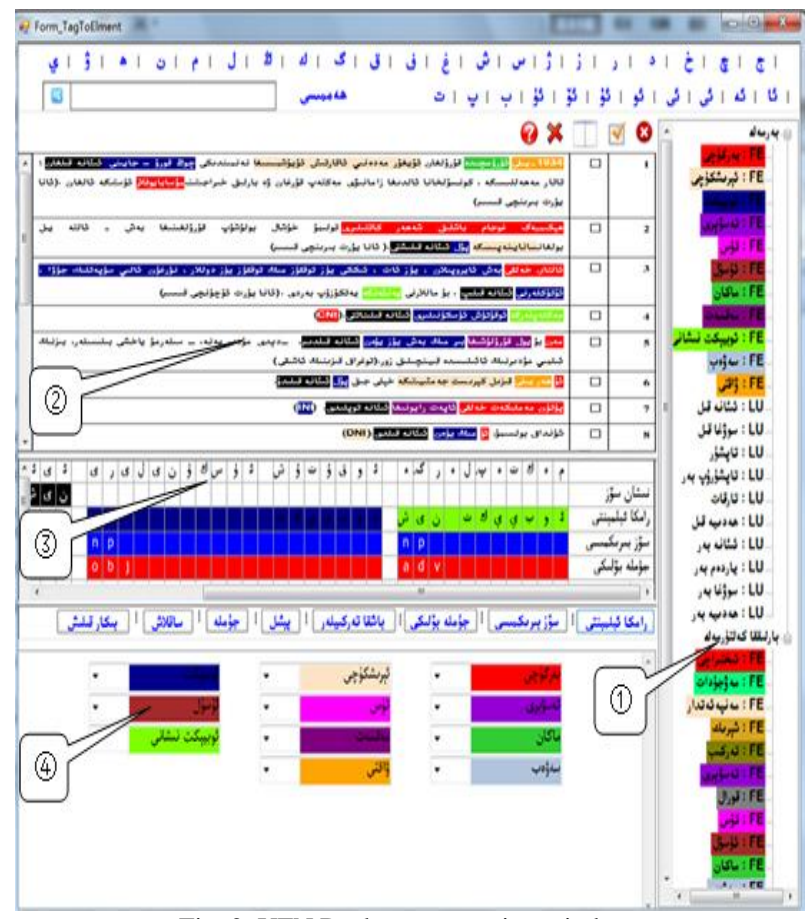

Fig. 2. UFN Desktop annotation window.

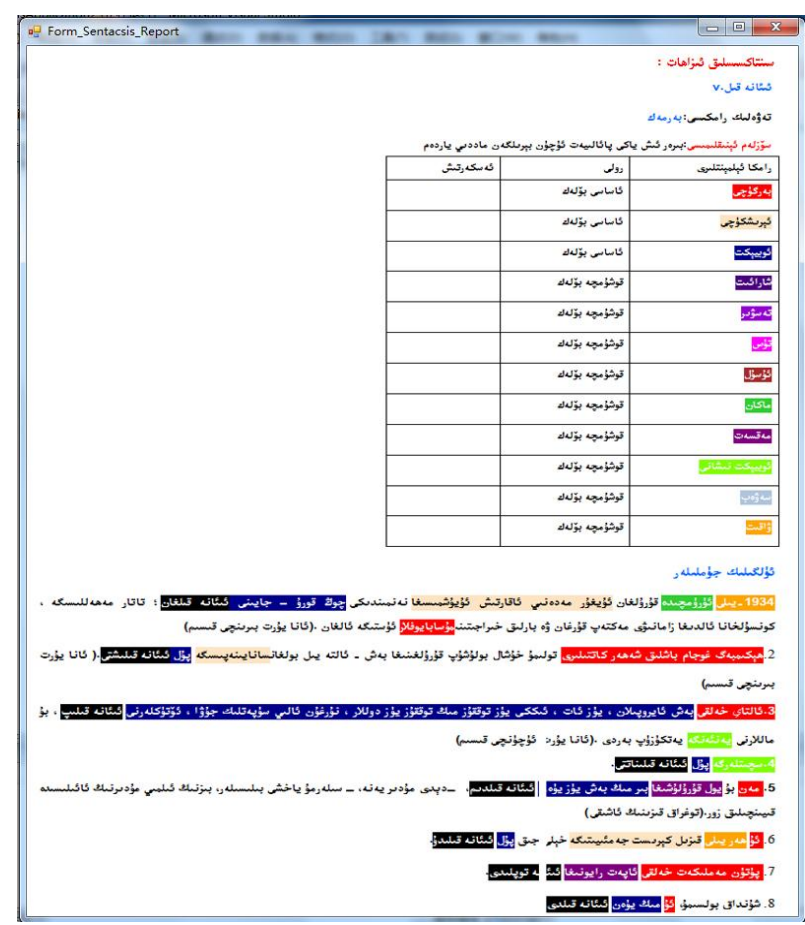

Fig. 3. Lexical unit report.

As displays in Fig. 3, the top of the report is a list of frame element ; this is called Frame Element table .The بمر مدك frame

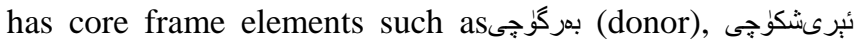
(recipient), ئوبيكت (theme), and has non-core frame elements such as شار ائىت (circumstance),

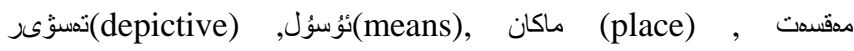

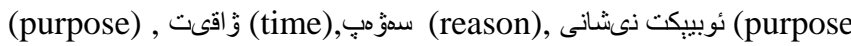


of theme), تؤس (manner). The bottom of the report is annotated sentence. The first table in Fig. 4 shows all of the ftame elements, the number of annotated examples and their syntactic realization. The second table in Fig. 4 shows the valence possibilities for

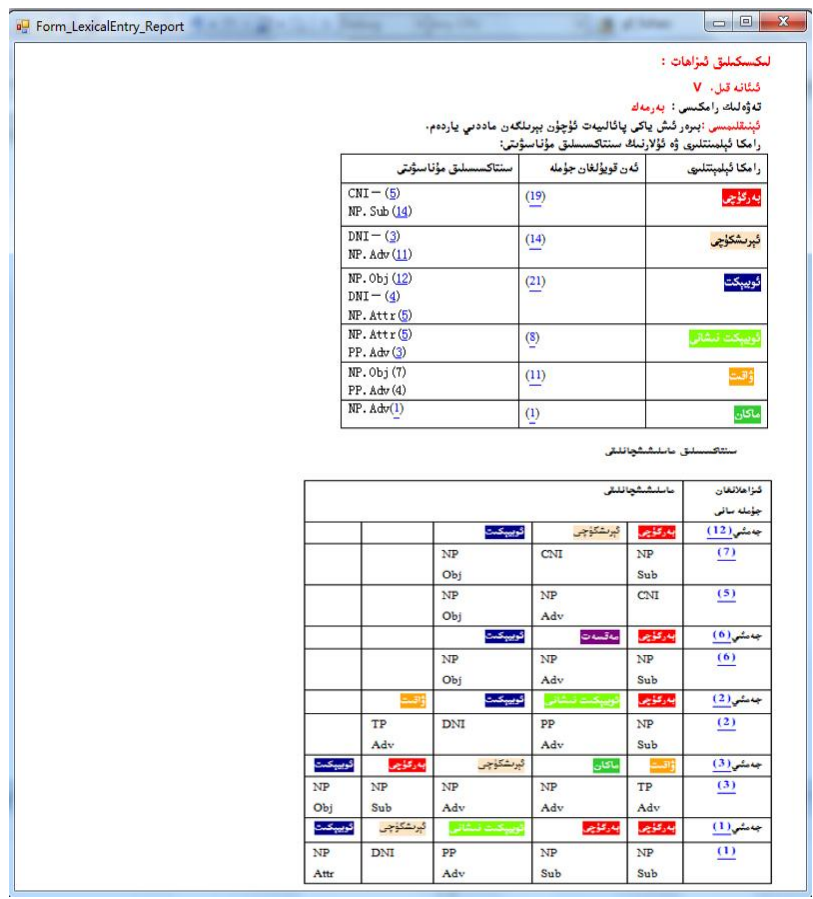

Fig. 4. Lexical extry report.

\section{CONCLUSION}

The Desktop annotation software has been designed for the UFN sentence annotation. The aim is to obtain various semantic and syntactic patterns which example sentences manifestation.

The UFN, by taking the large-scale Uyghur text language materials as the basis, and translating the Berkeley FrameNet project frames, and its corresponding information into equivalent frames in the Uyghur FrameNet. So far, approximately 402 frames have been constructed, with more than 4252 lexical unit, including verb, noun, adjective, covers broad semantics domain, annotated more than 18500 sentences.

There are still some shortcomings in the design, and we are adding some Shortcuts and more linguistic information for convenience of annotator. Our next step is using this UFN Desktop tool to build systematical and comprehensive corpora, and actually realize the Uyghur automatic semantic role labeling and question answering.

\section{REFERENCES}

[1] L. P. You, "A research on the construction of Chinese FrameNet," Ph.D. dissertation, Shanghai NormalUniversity, Shanghai, China, 2006.

[2] A Kuerban, W. Kuerban, and N. Abdurusul, "Research on Uyghur Framenet description system," in Proc. 2009 Oriental COCOSDA International Conference on Speech Database and Assessments, Urumqi, IEEE press, pp. 160-163, 2009.

[3] C. J. Fillmore, M. R. L. Petruck, J. Ruppenhofer, and A. Wright, "FrameNet in action: The case of attaching," International Journal of Lexicography, vol. 16, pp. 297-332, 2003.

[4] A. Kuerban, W. Kuerban, and D. Y. Fang, "Conceptual design of Uyghur FrameNet," Journal of Chinese Information Processing, vol. 27, pp. 41-46, March 2013.

[5] A. Kuerban, W. Kuerban, and T. Yibulayin, "A study of part of speech classification and tagsets of Uyghur language for information processing(I)," Jorunal of Xinjiang University(Natural Science Edition), vol. 26, pp. 476-481, Nov. 2009.

[6] A. Kuerban, W. Kuerban, and T. Yibulayin, "A study of part of speech classification and tagsets of Uyghur language for information processing(II)," Journal of Xinjiang University(Natural Science Edition), vol. 27, pp. 106-116, Feb. 2010.

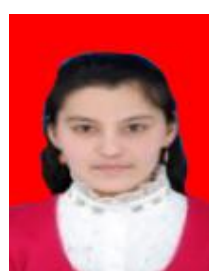

processing.

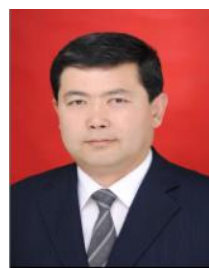

Alifu Kuerban was born in Korla, Xinjiang, China in 1967. He earned his master's degree in software engineering, Institute of Software Engineering of University of Electronic Science and Technology of China, Chengdu, China, in 2011. From 1993 to 2012, he worked as a lecturer in Xinjiang University, a professor from 2012-present. His research areas: ethnic minority language processing, database application.

So far he has published more than 20 articles. In 2008 he won the second prize of the "Science and Technology Progress", China; in 2009 won "Excellent Teacher" in Xinjiang Uyghur autonomous region, China. 